\title{
Malignant Pleural Mesothelioma Presenting as Achalasia
}

\author{
Hiroyuki Seki, Kazunori Matsumoto, Keiji Ohmura, Yukihiko Fukushi, Toshiaki FujtTa, \\ Hiroki KiriYama, Hiroshi TaKada, Kazuo KamiI, Haruhiko TeradA* and Yoshiomi Mori**
}

\begin{abstract}
A 65-year-old man with an occupational history of asbestos exposure developed dysphagia and vomiting. Clinical examinations at onset revealed a dilated esophagus with smooth narrowing at the gastroesophageal junction and no apparent tumor in and around the esophagus. Achalasia was suspected. Dysphagia progressed gradually and examinations performed three months after the onset disclosed a tumor in the pleural and the peritoneal cavitieis. At laparotomy, the tumor extended from the pleural cavity into the peritoneal cavity. Histological examination of the biopsied specimen demonstrated malignant mesothelioma. We report the first case of malignant pleural mesothelioma presenting as achalasia.
\end{abstract}

(Internal Medicine 33: 624-627, 1994)

Key words: asbestos exposure, dysphagia, pleural effusion, dilated esophagus, hyaluronic acid

\section{Introduction}

Malignant mesothelioma is a relatively rare tumor of mesodermal origin which arises in the pleural, pericardial or peritoneal cavity. Asbestos exposure is responsible in the majority of this type of tumor. In patients with malignant pleural mesothelioma, the most common presenting symptoms are chest pain and dyspnea, and a pleural effusion frequently develops. In this report, we describe a case of malignant pleural mesothelioma in whom the initial symptoms and clinical findings were similar to those of achalasia.

\section{Case Report}

A 65-year-old man was admitted to the hospital in April 1992 because of dysphagia, vomiting and body weight loss. There was an occupational history of exposure to asbestos. The patient had been treated for hypertension and asbestosis at another hospital since 1958. He developed dysphagia and vomiting about one month before admission. As barium esophagram revealed dilatation of the esophagus, achalasia was suspected. He was referred to the hospital for further evaluation and treatment.

On admission the body temperature was $36.6^{\circ} \mathrm{C}$ and the pulse rate was $78 / \mathrm{min}$. The blood pressure was $140 / 88 \mathrm{mmHg}$. Physical examination was negative except that he was thin and alert. Laboratory findings are listed in Table 1. Leukocytosis and an elevated erythrocyte sedimentation rate were found.
During the course of hospitalization, platelets increased to a maximum count of $710,000 / \mathrm{ml}$. Only CA- 125 was abnormal among the tumor markers examined. A chest $X$-ray film (Fig. 1) revealed diffuse granular opacities in both lungs, an unclear outline of pulmonary vessels and the lower part of the descending aorta, and a small amount of left pleural effusion. A computed tomography (CT) scan of the chest (Fig. 2) disclosed pleural thickening in addition to small nodularity in both lungs. Respiratory function tests (Table 2) showed respiratory restrictive ventilatory impairment. Blood gas analysis was normal. Barium esophagram (Fig. 3) showed smooth tapering of the dilated esophagus at the gastroesophageal junction. Endoscopic examinations (Fig. 4) revealed the dilated esophagus with

Table 1. Laboratory Findings on Admission

\begin{tabular}{lrlr}
\hline WBC & $13,100 / \mu \mathrm{l}$ & PLT & $39.3 \times 10^{4} / \mu \mathrm{l}$ \\
RBC & $465 \times 10^{4} / \mu \mathrm{l}$ & ESR & $73 \mathrm{~mm} / \mathrm{h}$ \\
Hb & $13.3 \mathrm{~g} / \mathrm{dl}$ & CRP & $(+)$ \\
& & & \\
GOT & $15 \mathrm{KU}$ & TP & $6.3 \mathrm{~g} / \mathrm{dl}$ \\
GPT & $4 \mathrm{KU}$ & Alb & $3.7 \mathrm{~g} / \mathrm{dl}$ \\
ALP & $5.8 \mathrm{KAU}$ & $\gamma$-gl & $1.2 \mathrm{~g} / \mathrm{dl}$ \\
LDH & $280 \mathrm{WU}$ & BUN & $11 \mathrm{mg} / \mathrm{dl}$ \\
$\gamma$-GTP & $17 \mathrm{U} / 1$ & Creat & $0.9 \mathrm{mg} / \mathrm{dl}$ \\
CH-E & $0.5 \Delta \mathrm{pH}$ & T-chol & $264 \mathrm{mg} / \mathrm{dl}$ \\
T-Bil & $0.88 \mathrm{mg} / \mathrm{dl}$ & CA125 & $118 \mathrm{U} / \mathrm{ml}(<35)$ \\
TTT & $1.0 \mathrm{MGU}$ & $\alpha-F P$ & $<10 \mathrm{ng} / \mathrm{ml}(<20)$ \\
ZTT & $11.8 \mathrm{KKU}$ & CEA & $1.2 \mathrm{ng} / \mathrm{ml}(<5)$ \\
\hline
\end{tabular}

From the Department of Gastroenterology and Hepatology, *the Department of Surgery and **the Department of Pathology, Koshigaya Hospital, Dokkyo University School of Medicine, Koshigaya

Received for publication January 28, 1994; Accepted for publication June 11, 1994

Reprint requests should be addressed to Dr. Hiroyuki Seki, the Department of Gastroenterology and Hepatology, Koshigaya Hospital, Dokkyo University School of Medicine, 2-1-50 Minami-koshigaya, Koshigaya, Saitama 343 


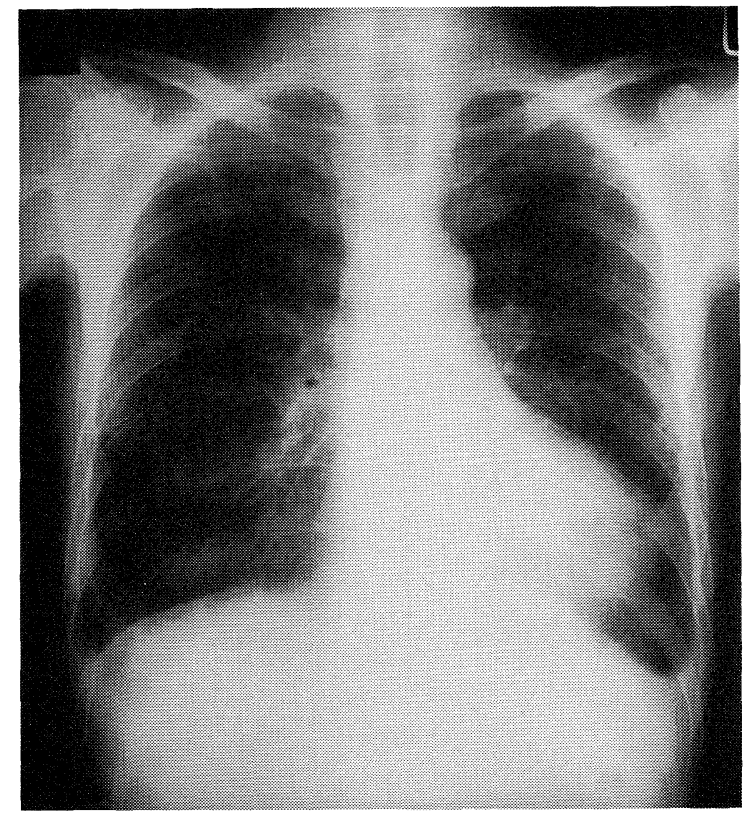

Fig. 1. Posteroanterior X-ray of the chest showing diffuse granular opacities in the lungs with a small amount of left pleural effusion.

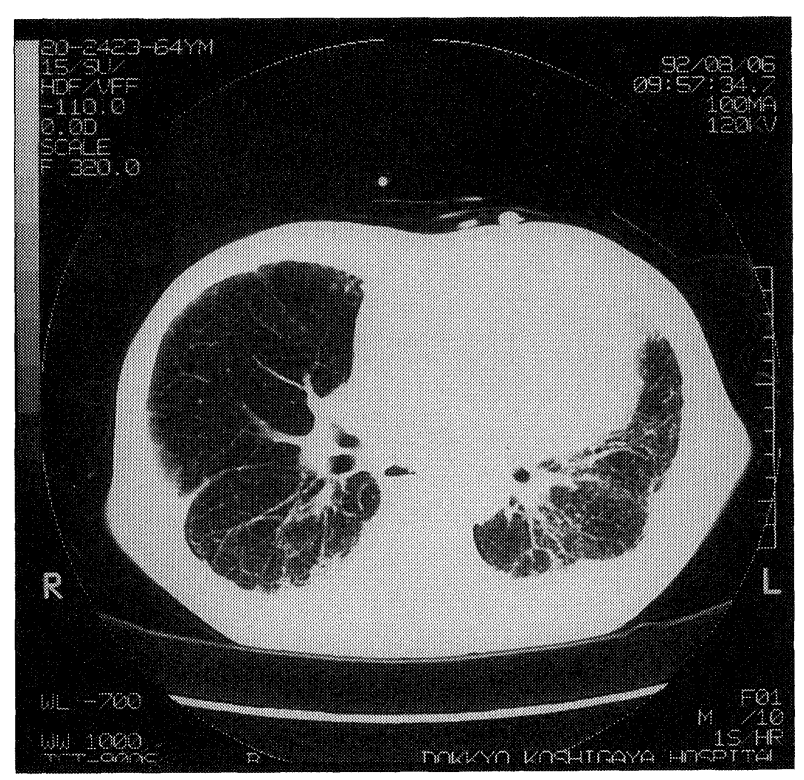

Fig. 2. CT scan of the chest revealing small nodularity in both lungs and pleural thickening.

Table 2. Respiratory Function Test and Blood Gas Analysis

\begin{tabular}{lrlr}
\hline $\mathrm{VC}$ & $1,420 \mathrm{ml}$ & $\mathrm{FEV}_{1.0}$ & $1,260 \mathrm{ml}$ \\
$\% \mathrm{VC}$ & $46.7 \%$ & $\mathrm{FEV}_{1.0} \%$ & $83.4 \%$ \\
$\mathrm{TV}$ & $390 \mathrm{ml}$ & & \\
$\mathrm{pH}$ & 7.440 & $\mathrm{BE}$ & $4.8 \mathrm{mEq} / \mathrm{l}$ \\
$\mathrm{PaO}_{2}$ & $100 \mathrm{mmHg}$ & $\mathrm{HCO}_{3}^{-}$ & $28.8 \mathrm{mEq} / \mathrm{l}$ \\
$\mathrm{PaCO}_{2}$ & $42 \mathrm{mmHg}$ & & \\
\hline
\end{tabular}

esophagitis and narrowing at the gastroesophageal junction. There were no malignant lesions in the esophagus and the stomach.

On manometric examination, the pressure of the lower esophageal sphincter was elevated to more than $60 \mathrm{mmHg}$. Swallowing decreased the pressure, while administration of anticholinergic drugs had little effect on the pressure. Manometric findings did not completely satisfy the criteria of achalasia. Dysphagia progressed gradually during hospitalization. An endoscope passed through the gastroesophageal junction without difficulty at the time of admission, whereas it could not be passed through at two months after admission. A CT scan of the chest and the upper abdomen (Fig. 5), performed after oral and intravenous administration of contrast material, demonstrated a mass which had not been recognized in the CT scan taken at the previous hospital about three months before and at our hospital on admission. The lower esophagus and the descending aorta were involved in the tumor.

Digital subtraction angiography (DSA) of the aorta (Fig. 6) disclosed a hypervascular tumor in the pleural cavity and a rightward deflection of the descending aorta. Sagittal magnetic resonance imaging (MRI) of the upper body revealed that the tumor extended from the pleural cavity into the peritoneal cavity. Cytologic examinations of the pleural effusion were negative. Hyaluronic acid in the pleural effusion was high at a concentration of more than $15 \mu \mathrm{g} / \mathrm{ml}$ (normal range: $2.5-5.5 \mu \mathrm{g}$ / $\mathrm{ml}$ ). We made a tentative diagnosis of malignant mesothelioma.

The patient underwent an operation because of inability to

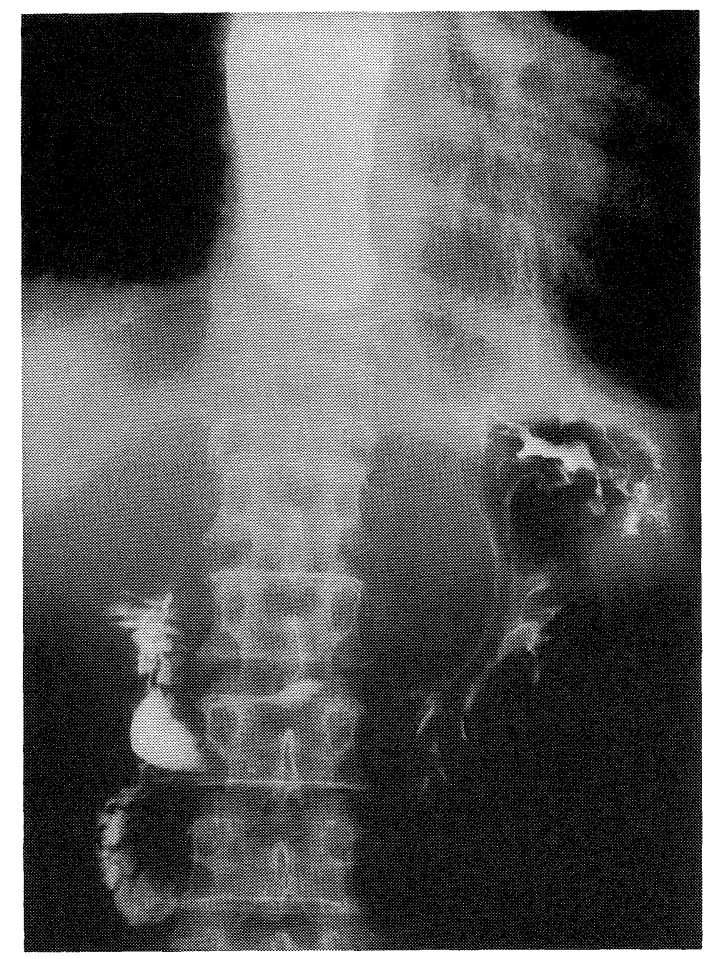

Fig. 3. Barium esophagram disclosing dilatation of the esophagus. 


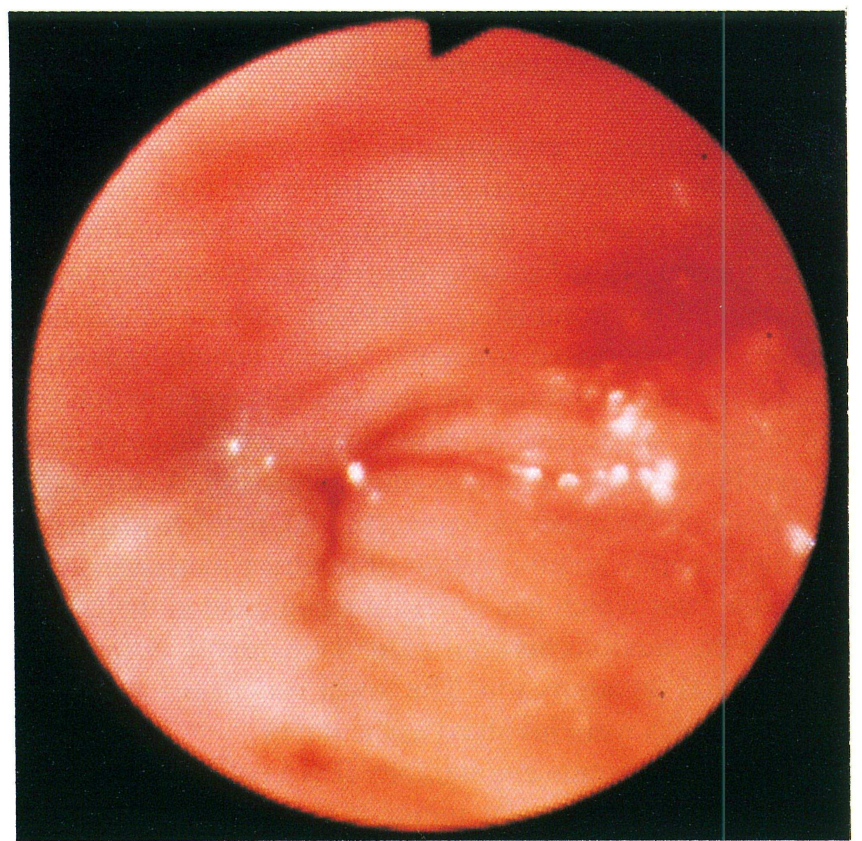

Fig. 4. Endoscopic picture of the esophagus showing stenosis at the gastroesophageal junction.

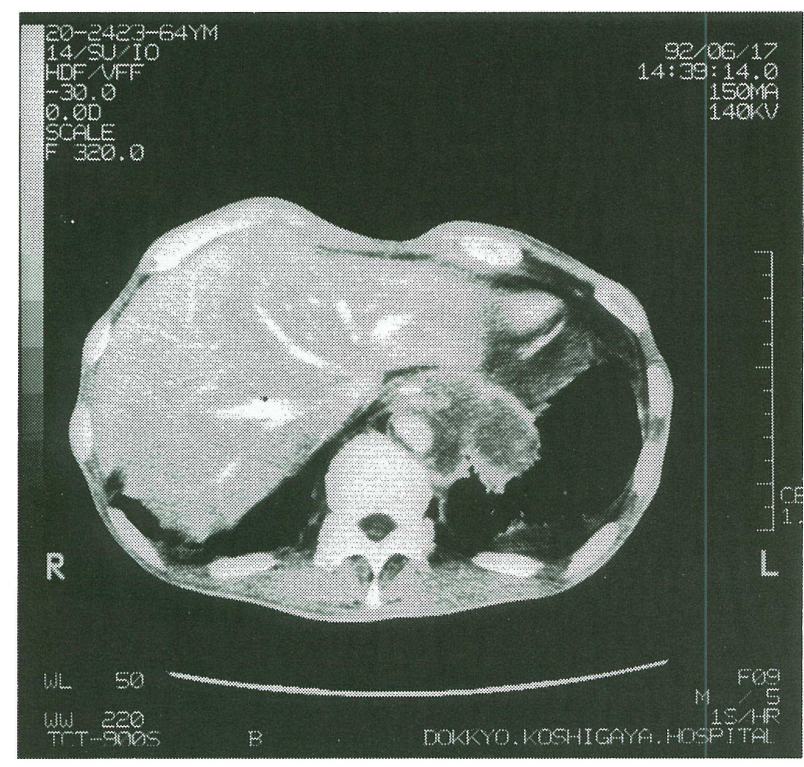

Fig. 5. CT scan of the upper abdomen taken after oral and intravenous administration of contrast medium disclosing a mass.

take a meal. At laparotomy, the tumor invaded the diaphragm and extended to the peritoneal cavity including the back of the pancreas and the left lobe of the liver. The tumor involved the lower portion of the esophagus.

As complete resection of the tumor was impossible, some palliative procedures were carried out and biopsy specimens were taken for pathological examination. Hematoxylin and $\operatorname{eosin}(\mathrm{HE}$ ) stain of biopsied specimens (Fig. 7) revealed epithelial

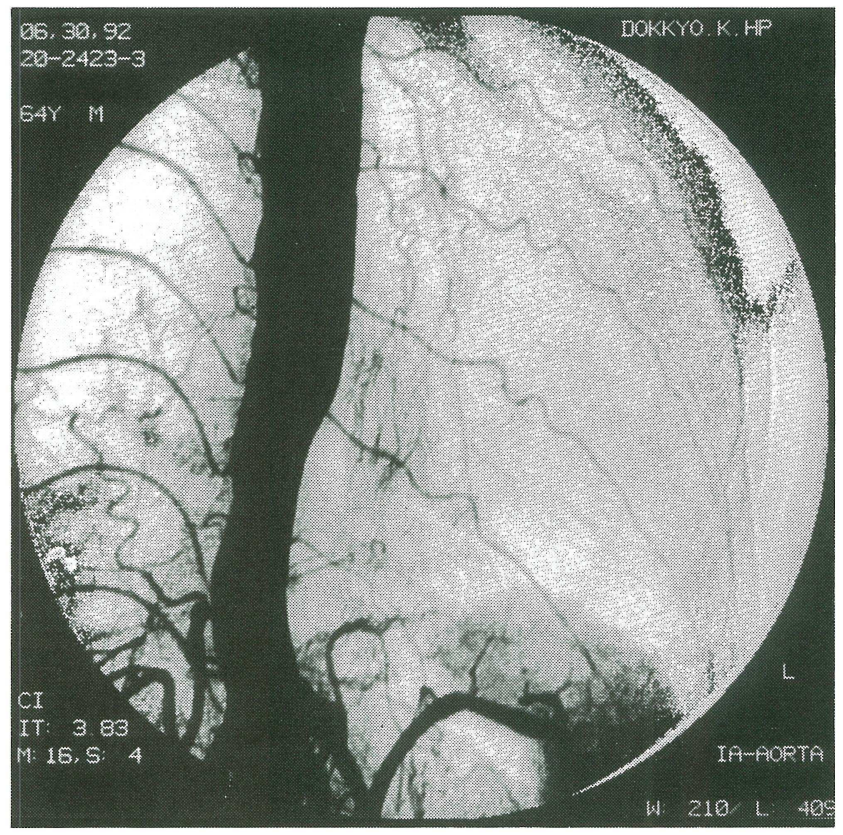

Fig. 6. DSA of the aorta demonstrating a hypervascular tumor and a rightward deflection of the descending aorta.

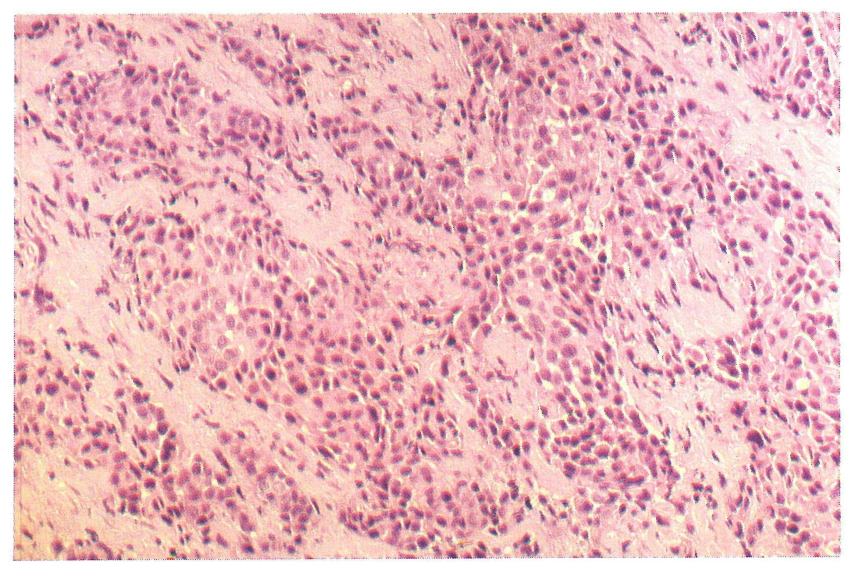

Fig. 7. Microscopic picture of the tumor $(\mathrm{HE}, \times 200)$.

tumor cells proliferating into the glandular structure. On colloidal iron stain (Fig. 8), tumor cells stained blue but did not stain after treatment with hyaluronidase. Pathological diagnosis of malignant mesothelioma was made. He died six months after the onset of symptoms.

\section{Discussion}

Malignant mesothelioma is relatively rare, but it is an increasingly recognized tumor of mesodermal origin which arises from the pleura, the peritoneum or less frequently the pericardium. Wagner et al first reported the close association of asbestos exposure and malignant mesothelioma in 1960 (1). It 


\section{Malignant Mesothelioma with Achalasia}
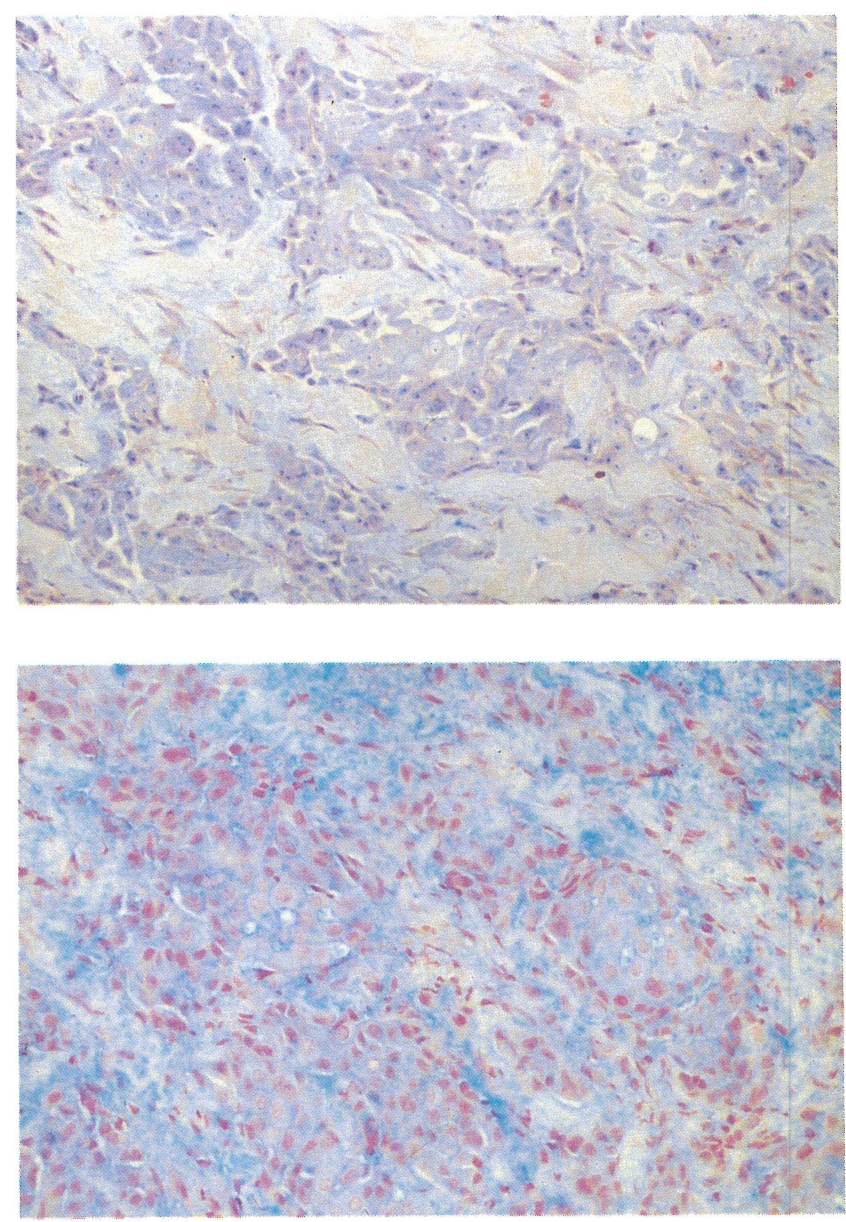

Fig. 8. Colloidal iron staining of the tumor with (upper) and without (lower) treatment with hyaluronidase $(\times 200)$.

is now generally accepted that asbestos is the most important etiologic agent of malignant mesothelioma (2-5). The latent period between asbestos exposure and development of malignant mesothelioma usually exceeds 20 years and sometimes may be 60 years (3). The most common presenting symptoms of malignant pleural mesothelioma are chest pain and dyspnea (5). Chest radiography frequently reveals a pleural effusion (4, 5). In the present patient, there was an occupational history of asbestos exposure and the latent period was about 35 years. The patient developed dysphagia and vomiting as initial symptoms. Barium esophagram and endoscopy revealed the dilated esophagus with stenosis at the gastroesophageal junction. Although we made every effort to rule out mechanical stenosis due to malignancy, we could not recognize any tumor in and around the digestive tracts at that time. However, we had a strong suspicion of malignancy, especially malignant pleural mesothelioma from the onset. Therefore, our tentative diagnosis was secondary achalasia due to yet undefined malignancy. We established the diagnosis of malignant pleural mesothelioma three months after the onset of symptoms, when the tumor was apparently recognized in the pleural and the peritoneal cavities. Thus, we concluded in retrospect that dilatation of the esophagus on the onset in our patient resulted from secondary achalasia due to malignant pleural mesothelioma. Secondary achalasia has been reported to occur in patients with a variety of malignancies (6). The most common malignancy is gastric carcinoma of the distal esophagus (6-8). To our knowledge, secondary achalasia due to malignant mesothelioma has not been reported so far. The pathogenesis of secondary achalasia remains unclear. Direct invasion of the distal esophageal myenteric plexus or neuropathic effect of an underlying malignancy has been thought to be the cause of secondary achalasia $(7,8)$. As malignant mesothelioma usually develops diffusely along parietal and viscera surfaces, direct invasion of the myenteric ganglia of the esophagus seems unlikely in our patient.

As to the pathologic diagnosis, HE stain of biopsied specimen showed epithelial malignant cells forming glandular structure, which resembled histological features of adenocarcinoma. On colloidal iron stain, tumor cells were stained blue, while the cells did not stain after treatment with hyaluronidase. This result indicates the presence of hyaluronic acid in the tumor cells. Furthermore, PAS stain with diastase digestion was negative. These pathologic findings are consistent with the diagnosis of malignant mesothelioma $(9,10)$.

The prognosis of malignant mesothelioma is extremely poor. The majority of the patients have been reported to die within the first year of the onset of symptoms $(4,11,12)$. The tumor in our patient grew rapidly and he died 6 months after the onset of symptoms. In conclusion, we describe here the first reported case of malignant pleural mesothelioma presenting as achalasia.

\section{References}

1) Wagner JC, Sleggs CA, Marchand P. Diffuse pleural mesothelioma and asbestos exposure in the North Western Cape Province. Br J Ind Med 17: $260,1960$.

2) Craighead JE. Current pathogenetic concepts of diffuse malignant mesothelioma. Hum Pathol 18: 544, 1987.

3) Ferguson DA, Berry G, Jelihovsky T, et al. The Australian Mesothelioma Surveillance Program. 1979-1985. Med J Aust 147: 166, 1987.

4) Borow M, Conston A, Livornese L, et al. Mesothelioma following exposure to asbestos. A review of 72 cases. Chest 64: 641, 1973.

5) Solomons K. Malignant mesothelioma - clinical and epidemiological features. A report of 80 cases. S Afr Med J 66: 407, 1984.

6) Tucker HJ, Snape WJ Jr, Cohen S. Achalasia secondary to carcinoma: Manometric and clinical features. Ann Int Med 89: 315, 1978.

7) Kolodny M, Schrader ZR, Rubin W, et al. Esophageal achalasia probably due to gastric carcinoma. Ann Intern Med 69: 569, 1968.

8) Schulze KS, Gorseky CA, Jabbari M, et al. Esophageal achalasia associated with gastric carcinoma: lack of evidence for widespread plexus destruction. Can Med Assoc J 112: 857, 1975.

9) Dewar A, Valente M, Ring NP, et al. Pleural mesothelioma of epithelial type and pulmonary adenocarcinoma: An ultrastructural and cytochemical comparison. J Pathol 152: 309, 1987.

10) Warnock ML, Stoloff A, Thor A. Differentiation of adenocarcinoma of the lung from mesothelioma. Periodic acid Schiff, monoclonal antibodies B72.3, and Leu M1. Am J Pathol 133: 30, 1988.

11) Wolf KM, Piotrowski ZH, Engel JD, et al. Malignant mesothelioma with occupational and environmental asbestos exposure in an Illinois community hospital. Arch Int Med 147: 2145, 1987.

12) Harrison RN, Hibberd SC, Dadds JH. Malignant pleural mesothelioma at St. Mary's Hospital, Portsmauth - a review of 29 fatal cases. Postgrad Med J 59: 712, 1983. 